\title{
Serum and Blister Fluid Pharmacokinetics of Amikacin in Severe Burn Patients
}

\author{
Rong Hua ${ }^{1^{*}}$, Hongliang Xu1 ${ }^{1}$ Xinzhou Rong ${ }^{2}$, Ronghua Yang3 \\ ${ }^{1}$ Department of Surgery, Wuxi Xishan Hospital, Wuxi, China \\ ${ }^{2}$ The First Municipal Hospital of Guangzhou Burn Unit, Guangzhou, China \\ ${ }^{3}$ The First Municipal Hospital of Foshan Burn Unit, Foshan, China \\ Email: *hzr19810915@sohu.com
}

Received 17 October 2015; accepted 20 November 2015; published 23 November 2015

Copyright (C) 2015 by authors and Scientific Research Publishing Inc.

This work is licensed under the Creative Commons Attribution International License (CC BY). http://creativecommons.org/licenses/by/4.0/

(c) (i) Open Access

\begin{abstract}
Objective: To characterize amikacin pharmacokinetics in serum and in blister fluid of severe burn patients to guide optimal treatment timing. Methods: Patients $(\mathbf{N}=32)$ were divided into four groups based on amikacin administration timing and groups received drug minutes to hours after injury. In Groups A, B, C, and D, amikacin (400 mg, IV) was administered 3- 4, 10, 20 and 30 h post burn injury, respectively ( $\mathrm{N}=8$ for all groups). Next blister fluid and venous blood samples from 9 patients were obtained at $0,0.25,0.5,1,2,3,4,5,6$, and $7 \mathrm{~h}$ after drug infusion. Amikacin concentrations were measured with a fluorescent polarization immunoassay and pharmacokinetics was deduced using DAS3.2.5. Statistical analyses performed with SPSS13.0. Results: Compared with normal values, $t_{1 / 2 z}$ of amikacin from burn patients was shortened in serum but amikacin halflives in blister fluid was significantly greater than serum half-life values $(p<0.05)$. Groups $A$ and $B$ had greater pharmacokinetic values at each time point, and Group D did not achieve antibacterial concentrations of amikacin. Conclusion: Early amikacin administration in severe burn patients offers greater concentrations of drug in serum and blister fluids.
\end{abstract}

\section{Keywords}

Amikacin Pharmacokinetics, Blister Fluid, Serum, Severe Burn Patients

\section{Introduction}

Burns cause loss of the protective skin barrier and subsequent suppression of immune function [1]. Necrotic tissue remaining at the wound and exudation fluids also offer suitable bacterial environment which can lead to both

\footnotetext{
"Corresponding author.
} 
wound and generalized infections. Thus, antibiotics are commonly used early to treat severe burn patients. Our previous research suggests that antibiotics are retained in the third space after early and short-term use of potent antibiotics and an antibiotic barrier is reported to form in the subeschar tissue fluid (STF) [2] [3], but they were lack of systematic investigations of antibiotic pharmacokinetics in sera and blister fluid samples. Numerous pathophysiological changes occur due to burn injury, and these changes may alter the pharmacokinetic characteristics of antimicrobial agents such as bioavailability, protein binding, volume of distribution, and clearance [4]-[9]. Amikacin, a member of the semisynthetic aminoglycoside family is a commonly used antimicrobial agent in burn units with broad spectrum antibacterial activity against pathogens, especially for intestinal tract Gram-negative bacteria and Pseudomonas aeruginosa [10]. Our objective was to characterize amikacin pharmacokinetics in serum and in blister fluid of severe burn patients to guide optimal treatment timing for this patient group.

\section{Materials and Methods}

\subsection{Study Design}

This was a prospective, open-labelled, non-randomized study of amikacin that was approved by the institutional review board of the hospital where the study was performed. Written informed consent was obtained from all patients or their legally designated representatives prior to study entry.

\subsection{Antibacterial Agent}

Amikacin sulfate (quantitate) was provided by Jin Ling Drug Manufacturer (Nan Jing, batch \# D60901, 2 ml: $200 \mathrm{mg})$.

\subsection{Instrumentation}

A Tdx immuno-analyzer was purchased from Abbott Chicago, IL). A table-top, high-speed centrifuge was from Guangzhou Medical Instrument Company Guangzhou, Guangdong Province). A precise transferpettor was also from Guangzhou Medical Instrument Company. A standard amikacin kit and a quality control kit were produced from Abbott (Chicago, IL). Amikacin was used at a concentration of $0-50 \mathrm{~g} / \mathrm{ml}$ and samples were analyzed by fluorescent polarization immunoassay (FPIA) using a Tdx immuno-analyzer.

\subsection{Patient Selection}

The study group included adult patients admitted to the First Municipal Hospital of Guangzhou Burn Unit, 2 to $30 \mathrm{~h}$ after burn injuries, and patient selection criteria were as follows: $\geq 18$ years-of-age, thermal injuries involving $\geq 30 \%$ total body surface area (TBSA) and creatinine clearance (CLCR) of $\geq 50 \mathrm{ml} / \mathrm{min}$. Patients with a history of amikacin use in the previous 2 weeks, or those receiving any drugs capable of interfering with the pharmacokinetics of amikacin were excluded from the study. Complete medical histories and physical examinations were obtained for each enrolled subject, and laboratory data for serum chemistries and hematology profiles were obtained and reviewed prior to sample collection for pharmacokinetic analysis. All subjects were monitored for adverse effects of amikacin throughout the duration of the study.

\subsection{Patients}

Severely burned patients with thermal injuries to $\geq 30 \%$ of the TBSA (TBSA; $N=32$, 22 males) were enrolled in this study, but only 9 patients donated blood (Table 1 and Table 2) depict their characteristics.

\subsection{Drug Administration}

Patients $(\mathrm{N}=32)$ were divided into four groups $(\mathrm{N}=8)$ based on amikacin administration timing and groups received drug minutes to hours after injury, all groups had second and third degree burns. For custom and security considerations, in Groups A, B, C, and D, amikacin (400 mg, IV, using a programmable pump) was administered $3-4,10,20$ and $30 \mathrm{~h}$ post burn injury, respectively. Next, blister fluid and venous blood samples were obtained at $0,0.5,1,2,3,4,5,6$, and $7 \mathrm{~h}$ after drug infusion. Blister fluid was collected from intact blisters 
Table 1. Patient demographics.

\begin{tabular}{cccccc}
\hline Group & Age (years) & Weight (kg) & TBSAB (\%) & 2 burn (\%) & 3 burn (\%) \\
\hline A & $30.25 \pm 8.08$ & $57.88 \pm 6.31$ & $55.63 \pm 14.50$ & $33.13 \pm 7.99$ & $22.25 \pm 7.07$ \\
B & $27.63 \pm 8.68$ & $60 \pm 7.54$ & $51.13 \pm 23.30$ & $29.38 \pm 14.25$ & $21.75 \pm 9.35$ \\
C & $32.63 \pm 6.5$ & $60.75 \pm 6.8$ & $57.5 \pm 19.64$ & $36.63 \pm 11.48$ & $21.88 \pm 8.43$ \\
D & $34.5 \pm 8.62$ & $63.13 \pm 7.53$ & $50.38 \pm 18.7$ & $28.75 \pm 11.57$ & $21.63 \pm 7.35$ \\
\hline
\end{tabular}

Table 2. Individual patient data.

\begin{tabular}{cccccccc}
\hline Patient \# & Sex & Age (years) & Weight (kg) & TBSAB (\%) & 2 burn (\%) & 3 burn (\%) & Hours postburn (h) \\
\hline 1 & Female & 19 & 55 & 65 & 35 & 30 & 4 \\
2 & Male & 30 & 65 & 60 & 40 & 20 & 20 \\
3 & Female & 30 & 48 & 40 & 25 & 15 & 20 \\
4 & Male & 21 & 60 & 30 & 20 & 10 & 10 \\
5 & Female & 28 & 50 & 30 & 15 & 15 & 3 \\
6 & Male & 30 & 60 & 60 & 35 & 25 & 10 \\
7 & Female & 44 & 50 & 35 & 20 & 15 & 30 \\
8 & Female & 18 & 50 & 40 & 25 & 15 & 30 \\
9 & male & 35 & 65 & 40 & 20 & 20 & 14.56 \\
Mean & & 28.33 & 55.89 & 44.44 & 26.11 & 18.33 & 10.81 \\
\hline
\end{tabular}

(1 - $2 \mathrm{ml})$. All patients received standard resuscitation and burn wound care. Amikacin concentrations were measured with a FPIA and pharmacokinetics were deduced using DAS3.2.5. Statistical analyses performed with SPSS13.0.

\subsection{Sample Collection and Storage}

Blister fluid was collected with a syringe at $0,0.25,0.5,1,2,3,4,5,6$, and $7 \mathrm{~h}$ after drug infusion and blood was sampled on the same time line. Blood samples were centrifuged at $4000 \times \mathrm{g}$ for $10 \mathrm{~min}$ and then both sera and fluid were placed into polypropylene containers and frozen at $-70^{\circ} \mathrm{C}$ and thawed prior to use.

\subsection{Sample Assay}

Amikacin concentrations in sera and blister fluid samples were measured using a FPIA and drug pharmacokinetics was measured using DAS3.2.5. Drug analysis system statistical software was used to analyze data using new drug statistical processing software.

\subsection{Statistical Analysis}

Amikacin pharmacokinetic data were statistically analyzed using the leading free statistical software package, SPSS13.0, by Softonic. Groups were compared using a two-sided Student's t test $(P<0.05$ was considered statistically significant).

\section{Results}

Amikacin in the serum peaked after infusion and compared to healthy patients, burn patients had shorter drug half-lives. Figure 1, Table 3 and Table 4 depict these data. Amikacin was higher in the blister fluid of groups A and $\mathrm{B}$ at each time point $(P<0.05)$. Figure 2 and Table 5 depict these data. Compared to groups $\mathrm{C}$ and $\mathrm{D}$ groups, groups A and B had higher AUCs and shorter drug half-lives $(P<0.05)$. Table 6 depicts these data. 
Table 3. Amikacin concentration $(\mathrm{mg} / \mathrm{L})$ in serum $(\mathrm{n}=9)$.

\begin{tabular}{|c|c|c|c|c|c|c|c|c|c|c|}
\hline \multirow{2}{*}{ Patient } & \multicolumn{10}{|c|}{ Time (h) } \\
\hline & 0 & 0.25 & 0.5 & 1 & 2 & 3 & 4 & 5 & 6 & 7 \\
\hline 1 & 38.25 & 32.23 & 17.39 & 12.34 & 8.49 & 5.62 & 4.65 & 2.23 & 1.86 & 1.32 \\
\hline 2 & 42.09 & 29.71 & 21.97 & 13.48 & 7.28 & 6.08 & 3.64 & 1.62 & 1.42 & 0.86 \\
\hline 3 & 43.86 & 27.75 & 22.95 & 14.65 & 8.51 & 5.85 & 2.61 & 1.73 & 1.35 & 0.76 \\
\hline 4 & 34.41 & 22.15 & 18.09 & 14.68 & 10.29 & 6.89 & 5.17 & 2.41 & 1.67 & 1.53 \\
\hline 5 & 32.03 & 20.22 & 17.44 & 13.46 & 9.99 & 6.03 & 5.29 & 2.24 & 1.53 & 1.12 \\
\hline 6 & 41.43 & 24.44 & 19.42 & 13.62 & 6.93 & 5.29 & 3.21 & 2.27 & 1.41 & 0.43 \\
\hline 7 & 36.09 & 24.18 & 16.79 & 12.61 & 7.10 & 5.04 & 3.36 & 2.33 & 1.21 & 0.25 \\
\hline 8 & 39.78 & 24.56 & 18.78 & 12.52 & 8.88 & 6.26 & 3.65 & 2.35 & 1.78 & 1.13 \\
\hline 9 & 48.43 & 28.01 & 21.00 & 15.83 & 10.79 & 7.86 & 5.64 & 3.36 & 2.23 & 1.38 \\
\hline Mean & 39.60 & 25.92 & 19.31 & 13.69 & 8.70 & 6.10 & 4.14 & 2.28 & 1.61 & 0.98 \\
\hline SD & 5.05 & 3.80 & 2.19 & 1.17 & 1.43 & 0.85 & 1.07 & 0.49 & 0.31 & 0.44 \\
\hline
\end{tabular}

Table 4. Serum pharmacokinetics for amikacin.

\begin{tabular}{ccccccccc}
\hline Patient & $\begin{array}{c}\mathbf{A U C}(\mathbf{0 - t )} \\
\mathbf{m g} / \mathbf{L} * \mathbf{h}\end{array}$ & $\begin{array}{c}\mathbf{A U C}(\mathbf{0 - \infty}) \\
\mathbf{m g} / \mathbf{L} * \mathbf{h}\end{array}$ & $\begin{array}{c}\mathbf{A U M C}(\mathbf{0}-\infty) \\
\mathbf{h} * \mathbf{h} * \mathbf{m g} \mathbf{L}\end{array}$ & $\begin{array}{c}\mathbf{M R T}_{(\mathbf{0}-\infty)} \\
\mathbf{h}\end{array}$ & $\begin{array}{c}\mathbf{V R T}_{(\mathbf{0}-\infty)} \\
\mathbf{h}^{\mathbf{2}}\end{array}$ & $\begin{array}{c}\mathbf{t}_{\mathbf{1} / 2 \mathbf{z}} \\
\mathbf{h}\end{array}$ & $\begin{array}{c}\mathbf{V z} \\
\mathbf{L}\end{array}$ & $\begin{array}{c}\mathbf{C L z} \\
\mathbf{L} / \mathbf{h}\end{array}$ \\
\hline 1 & 51.85 & 54.86 & 122.00 & 2.22 & 6.08 & 1.63 & 17.15 & 7.29 \\
2 & 51.26 & 53.06 & 99.54 & 1.88 & 4.48 & 1.45 & 15.72 & 7.54 \\
3 & 52.21 & 54.06 & 98.91 & 1.83 & 4.57 & 1.69 & 17.99 & 7.40 \\
4 & 54.64 & 56.21 & 118.96 & 2.12 & 4.11 & 1.23 & 12.59 & 7.12 \\
5 & 51.16 & 54.36 & 129.76 & 2.39 & 6.59 & 2.00 & 21.23 & 7.36 \\
6 & 47.90 & 50.23 & 101.20 & 2.02 & 5.44 & 1.69 & 19.36 & 7.96 \\
7 & 45.27 & 46.76 & 90.05 & 1.93 & 4.25 & 1.36 & 16.75 & 8.55 \\
8 & 50.82 & 53.71 & 119.67 & 2.23 & 6.14 & 1.79 & 19.23 & 7.45 \\
9 & 63.14 & 66.10 & 147.36 & 2.23 & 5.27 & 1.48 & 12.92 & 6.05 \\
Mean & 52.03 & 54.37 & 114.16 & 2.09 & 5.22 & 1.59 & 16.99 & 7.41 \\
SD & 4.95 & 5.23 & 18.22 & 0.19 & 0.91 & 0.24 & 2.90 & 0.67 \\
\hline
\end{tabular}

Table 5. Amikacin concentration $(\mathrm{mg} / \mathrm{L})$ in patient groups $(\mathrm{n}=8)$ Mean \pm SD.

\begin{tabular}{cccccccccc}
\hline Group & $\mathbf{0 . 2 5} \mathbf{h}$ & $\mathbf{0 . 5} \mathbf{h}$ & $\mathbf{1 ~ h}$ & $\mathbf{2} \mathbf{h}$ & $\mathbf{3 ~ h}$ & $\mathbf{4} \mathbf{h}$ & $\mathbf{5 h}$ & $\mathbf{6 ~ h}$ & $\mathbf{7 h}$ \\
\hline A & $4.55 \pm 1.40$ & $8.94 \pm 1.77$ & $12.43 \pm 1.36$ & $10.73 \pm 2.82$ & $8.44 \pm 1.74$ & $6.57 \pm 2.16$ & $4.89 \pm 1.62$ & $3.65 \pm 1.15$ & $2.13 \pm 0.50$ \\
B & $4.75 \pm 1.35$ & $6.44 \pm 1.53$ & $9.12 \pm 1.47$ & $9.46 \pm 0.97$ & $7.17 \pm 0.64$ & $6.08 \pm 0.63$ & $4.43 \pm 0.88$ & $3.09 \pm 0.78$ & $1.98 \pm 0.52$ \\
C & $1.36 \pm 0.28$ & $2.58 \pm 0.55$ & $4.67 \pm 0.59$ & $5.88 \pm 0.80$ & $4.90 \pm 0.59$ & $3.82 \pm 0.62$ & $2.88 \pm 0.46$ & $2.13 \pm 0.25$ & $1.41 \pm 0.45$ \\
D & $1.24 \pm 0.55$ & $1.86 \pm 0.44$ & $3.27 \pm 0.40$ & $2.90 \pm 0.20$ & $2.42 \pm 0.14$ & $1.92 \pm 0.13$ & $1.49 \pm 0.16$ & $1.08 \pm 0.29$ & $0.87 \pm 0.28$ \\
\hline
\end{tabular}

\section{Discussion}

Burn wounds are optimal injuries for bacterial invasion and infection and sepsis are leading causes of death in severe burn patients. Thus, preventing wound infection or reducing bacterial toxin absorption is critical for preventing generalized infections, suggesting that rational antibiotic use is necessary for severe burn patient treatment. 
Table 6. Pharmacokinetic parameters of amikacin in patient groups $(\mathrm{n}=8)$ Mean \pm SD.

\begin{tabular}{|c|c|c|c|c|c|c|c|c|c|c|}
\hline Patient & $\operatorname{AUC}_{(0-\mathrm{t})}$ & $\operatorname{AUC}_{(0-\infty)}$ & $\operatorname{AUMC}_{(0-\infty)}$ & $\operatorname{MRT}_{(0-\infty)}$ & $\operatorname{VRT}_{(0-\infty)}$ & $\mathbf{t}_{1 / 2 \mathbf{z}}$ & $\mathbf{T}_{\max }$ & $\mathbf{V z}$ & CLz & $\mathrm{C}_{\max }$ \\
\hline Group & $\mathrm{mg} / \mathrm{L} * \mathbf{h}$ & $\mathrm{mg} / \mathrm{L} * \mathbf{h}$ & $\mathbf{h} * \mathbf{h} * \mathbf{m g} / \mathbf{L}$ & h & $\mathbf{h}^{2}$ & h & h & $\mathbf{L}$ & $\mathbf{L} / \mathbf{h}$ & $\mathrm{mg} / \mathrm{L}$ \\
\hline A & $49.15 \pm 10.22$ & $55.84 \pm 13.16$ & $205.17 \pm 69.50$ & $3.62 \pm 0.45$ & $9.05 \pm 3.63$ & $1.93 \pm 0.501$ & $1.25 \pm 0.46$ & $20.53 \pm 6.49$ & $7.44 \pm 1.361$ & $12.73 \pm 1.65$ \\
\hline B & $41.65 \pm 5.40$ & $46.73 \pm 7.15$ & $169.40 \pm 36.70$ & $3.60 \pm 0.28$ & $7.58 \pm 1.71$ & $1.72 \pm 0.241$ & $1.75 \pm 0.46$ & $21.43 \pm 2.44$ & $8.73 \pm 1.27$ & $9.70 \pm 1.20$ \\
\hline $\mathrm{C}$ & $25.12 \pm 2.66$ & $30.97 \pm 3.21$ & $142.20 \pm 34.81$ & $4.56 \pm 0.90$ & $14.45 \pm 8.51$ & $2.49 \pm 0.762$ & $2.13 \pm 0.354$ & $46.15 \pm 12.89$ & $13.06 \pm 1.60$ & $5.95 \pm 0.63$ \\
\hline $\mathrm{D}$ & $13.70 \pm 1.02$ & $18.07 \pm 3.86$ & $95.50 \pm 52.52$ & $5.00 \pm 1.592$ & $22.92 \pm 20.10$ & $03.05 \pm 1.341$ & $1.25 \pm 0.469$ & $93.97 \pm 20.99$ & $22.97 \pm 4.55$ & $3.37 \pm 0.25$ \\
\hline
\end{tabular}

Note: $\mathrm{t}_{1 / 2 z}$, elimination half-life; $\mathrm{Vz}$, apparent volume of distribution; AUC, area under the curve; CLz, clearance; $\mathrm{T}_{\max }$, drug concentration peak arrival time.

\section{Concentration-time curves}

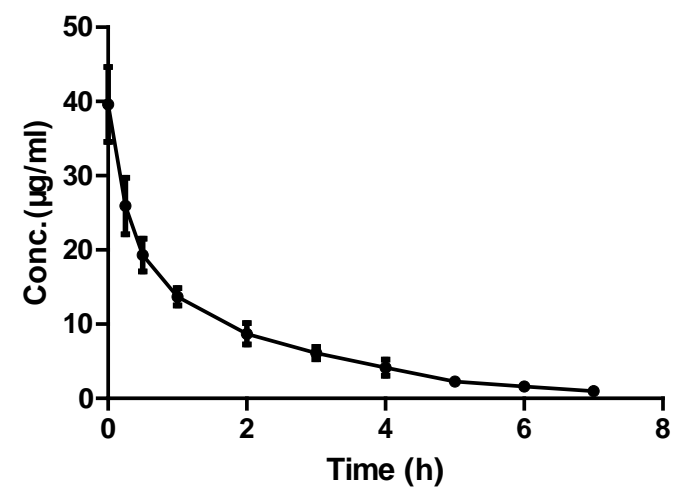

Figure 1. Concentration profiles for amikacin in serum after single infusions ( $n=9$, mean \pm S.E.M.).

\section{Concentration-time curves}

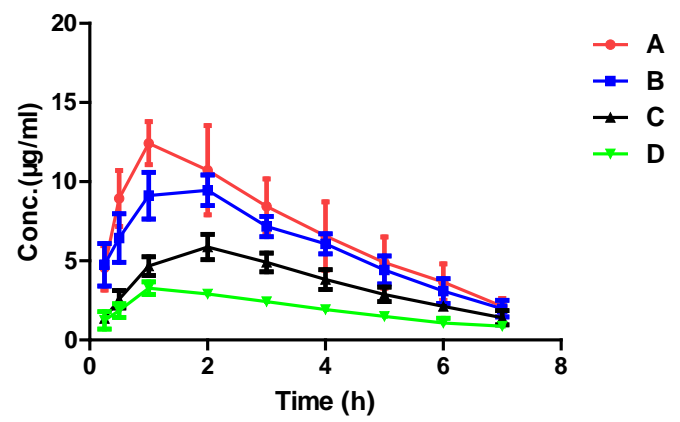

Figure 2. Concentration profiles for amikacin in blister fluid after single infusion, in In Groups A, B, C, and D, (n = 8, mean \pm S.E.M.).

Documenting the best antibiotic regimen for drug accumulation and sustained residence time within burn wounds was the focus of our work. Our data suggest that when amikacin was administered 3 - 4 h post burn (Group A), maximal drug concentration was reached in blister fluid at $1.25 \mathrm{~h}$ (Table 6) and this decreased after the infusion. Group B was similar (amikacin given $10 \mathrm{~h}$ post-burn) and amikacin peaked at $1.75 \mathrm{~h}$ (Table 6), which was significantly higher than serum concentrations measured at 3, 4, 5, 6, $7 \mathrm{~h}$ after infusion. From Groups $\mathrm{C}$ and $\mathrm{D}$, we observed that $\mathrm{C}_{\max }$ was low, significantly lower than Groups A and B Groups $(P<0.05)$ and Groups $\mathrm{A}$ and $\mathrm{B}$ had greater drug concentrations at each time point compared to Groups $\mathrm{C}$ and $\mathrm{D}(P<0.05$ or $P<$ 0.01), especially $1 \mathrm{~h}$ post-infusion (Table 5). Furthermore, the amikacin concentration in Groups A and B were higher than most $\mathrm{MIC}_{50}(\leq 5 \mathrm{~g} / \mathrm{ml})$ of common pathogenic bacteria [11], including Pseudomonas aeruginosa which is a common burn unit pathogen [12]. In contrast, amikacin concentrations in Groups C and D were less than $\mathrm{MIC}_{50}$ for common pathogenic bacteria [11]. Therefore, we suggest that giving amikacin within 10 of a 
burn injury is useful and may form an "antibiotic barrier" around the burn wound to prevent invasive infection, such as Staphylococcus aureus and Pseudomonas aeruginosa. Also, amikacin administration 20 or $30 \mathrm{~h}$ after a burn may not offer anti-infective effects. Thus, later amikacin administration may require higher doses.

In healthy young adults $\left(\mathrm{t}_{1 / 2 \mathrm{z}}=2.1+0.2 \mathrm{~h}\right)$ [13], the serum half-life of amikacin $\left(\mathrm{t}_{1 / 2 \mathrm{z}}=1.59+0.34 \mathrm{~h}\right)$ was significantly shortened in severe burn patients at early stages, a finding that was in agreement with data from Zaske's laboratory [14]. Charles's group [15] considered this to be related to increased glomerular filtration and renal clearance of the drug. Also, increased capillary permeability and rapid wound exudation and increased nonrenal clearance rates contribute to alterations of drug pharmacokinetics [5] [16].

In short, early administration of amikacin to severe burn patients increased the drug concentration in blister fluid and prolonged the drug half-life. This enabled blisters to form an "antibiotic barrier" around the wound surface to prevent invasive infections. These data strongly suggest early short-term antibiotic use to reduce overall doses administered during the patient hospital stay, which improves patient care and decreases healthcare costs.

\section{Acknowledgements}

We thank Tao Zhang, Xiaohua Huang, Qinghui Li, and Yuanming Su, all of them were responsible for the care and treatment of the patients. We thank Jianjian Liang and Kai Huang for technical help. They are both pharmacists.

\section{Conflicts of Interest and Source of Funding}

The authors have no conflict of interest. This study was funded by Science and Technology key projects in Wuxi city.

\section{References}

[1] Schwacha, M.G., Knöferl, M.W. and Chaudry, I.H. (2000) Does Burn Wound Excision after Thermal Injury Attenuate Subsequent Macrophage Hyperactivity and Immunosuppression? Shock, 14, 623-628. http://dx.doi.org/10.1097/00024382-200014060-00009

[2] Rong, X.Z., Zhang, W.Z. and Ren, J.L. (2001) A Clinical Observation of Early Short Term Use of Potent Antibiotics in Severely Burned Patients. Journal of Medical Colleges of PLA, 16, 252-254.

[3] Yang, R.H., Rong, X.Z., Hua, R., et al. (2009) Pharmacokinetics of Vancomycin and Amikacin in the Subeschar Tissue Fluid in Patients with Severe Burn. Burns, 35, 75-79. http://dx.doi.org/10.1016/j.burns.2008.05.016

[4] Boucher, B.A., Kuhl, D.A. and Hickerson, W.L. (1992) Pharmacokinetics of Systemically Administered Antibiotics in Patients with Thermal Injury. Clinical Infectious Diseases, 14, 458-463. http://dx.doi.org/10.1093/clinids/14.2.458

[5] Jaehde, U. and Sörgel, F. (1995) Clinical Pharmacokinetics in Patients with Burns. Clinical Pharmacokinetics, 29, 15-28. http://dx.doi.org/10.2165/00003088-199529010-00003

[6] Varela, J.E., Cohn, S.M., et al. (2000) Pharmacokinetics and Burn Eschar Penetration of Intravenous Ciprofloxacin in Patients with Major Thermal Injuries. Journal of Antimicrobial Chemotherapy, 45, 337-342. http://dx.doi.org/10.1093/jac/45.3.337

[7] Peck, M.D. and Ward, C.G. (1997) Burn Injury. In: Civetta, J.M., Taylor, R.W., Kirby, R.R., Eds., Critical Care, Lippincott-Raven Publishers, Philadelphia, 1265-1275.

[8] Sangil, J., Seunghoon, H., Jongtae, L., et al. (2014) Population Pharmacokinetic Analysis of Piperacillin in Burn Patients. Journal of Antimicrobial Chemotherapy, 58, 3744-3751. http://dx.doi.org/10.1128/AAC.02089-13

[9] Kevin, S.A., Matthew, P.R., Krista, L., et al. (2015) Colistin Pharmacokinetics in Burn Patients during Continuous Venovenous Hemofiltration. Journal of Antimicrobial Chemotherapy, 59, 46-52. http://dx.doi.org/10.1128/AAC.03783-14

[10] Tally, F.P., Louie, T.J., Weinstein, W.M., et al. (1975) Amikacin Therapy for Severe Gram-Negative SepsisEmphasis on Infections with Gentamicin-Resistant Organisms. Annals of Internal Medicine, 83, 484-488. http://dx.doi.org/10.7326/0003-4819-83-4-484

[11] Hu, B.J., Chen, X.H. and Ni, C.M. (1999) The Evaluation and the Result of 12 Antibiotics Sensitive to Nosocomial Infection Bacteria. Chin J Nosocomiol, 9, 118-120.

[12] Dou, Y., Zhang, Q. and Liao, Z.J. (2004) Investigation on the Drug Resistance of Pseudomonas aeruginosa in Our Burn Ward in the Past 11 Years. Chinese Journal of Burns, 20, 6-9. 
[13] Shi, Y.G., Zhang, Y.Y., Zhang, J., Wang, Z.P., Yu, J.C. and Wang, F. (2001) Pharmacokinetics of Antimicrobial Agents in the Elderly. Chinese Journal of Infection and Chemotherapy, 1, 3-6.

[14] Zaske, D.E., Sawchuk, R.J. and Strate, R.G. (1978) The Necessity of Increased Doses of Amikacin in Burn Patients. Surgery, 84, 603-608.

[15] Bonapace, C.R., White, R.L., Friedrich, L.V., Norcross, E.D. and Bosso, J.A. (1999) Pharmacokinetics of Cefepime in Patients with Thermal Burn Injury. Antimicrobial Agents and Chemotherapy, 43, 2848-2854.

[16] Bourget, P., Lesne-Hulin, A., Le Reveillé, R., Le Bever, H. and Carsin, H. (1996) Clinical Pharmacokinetics of Piperacillin-Tazabactam Combination in Patients with Major Burns and Signs of Infection. Antimicrobial Agents and Chemotherapy, 40, 139-145. 